Pacific

Journal of

Mathematics

ALTERNATING GROUPS AS MONODROMY GROUPS IN POSITIVE CHARACTERISTIC

Stefan WeWers and Irene I. Bouw

Volume 222 No. 1

November 2005 


\title{
ALTERNATING GROUPS AS MONODROMY GROUPS IN POSITIVE CHARACTERISTIC
}

\author{
STEFAN WEWERS AND IRENE I. BOUW
}

\begin{abstract}
Let $X$ be a generic curve of genus $\boldsymbol{g}$ defined over an algebraically closed field $k$ of characteristic $p \geq 0$. We show that for $\boldsymbol{n}$ sufficiently large there exists a tame rational map $f: X \rightarrow \mathbb{P}_{k}^{1}$ of degree $n$ with monodromy group $A_{n}$. This generalizes a result of Magaard and Völklein to positive characteristic.
\end{abstract}

\section{Introduction}

1.1. Let $k$ be an algebraically closed field of characteristic $p \geq 0$. We denote by $k_{0}\left(=\mathbb{Q}\right.$ or $\left.\mathbb{F}_{p}\right)$ the prime field of $k$. For $g \geq 0$, we write $M_{g}$ for the (coarse) moduli space of curves of genus $g$ in characteristic $p$. This is a smooth, quasi-projective and geometrically irreducible variety over $k_{0}$; see [Deligne and Mumford 1969]. Its dimension is $3 g-3$ if $g \geq 2$, and $g$ otherwise.

Let $X$ be a smooth projective curve of genus $g$ over $k$. It corresponds to a $k$ rational point $x:$ Spec $k \rightarrow M_{g}$. We say that $X$ is generic if the image of $x$ is Zariski dense in $\mu_{g}$.

Let $f: X \rightarrow \mathbb{P}_{k}^{1}$ be a nonconstant rational function on $X$. We say that $f$ is tame if the extension of function fields $k(X) / k(f)$ is separable and at most tamely ramified. The degree of the extension $k(X) / k(f)$ is called the degree of $f$. The Galois group of the Galois closure of $k(X) / k(f)$ is called the monodromy group of $f$. The aim of this note is to prove the following theorem.

Theorem 1. Let $g \geq 0$ and $n \geq 3$. Let $X$ be a generic curve of genus $g$, defined over an algebraically closed field $k$ of characteristic $p \geq 0$. Then the curve $X$ admits $a$ tame rational function $f: X \rightarrow \mathbb{P}_{k}^{1}$ of degree $n$ with alternating monodromy group, in each of the following cases:

(a) $p \neq 2,3$ and $n \geq \max (g+3,2 g+1)$.

(b) $p=2, n \geq \max (g+3,2 g+1)$ and $n+g$ is odd.

(c) $p=2, n \geq \max (g+6,2 g+3)$ and $n+g$ is even.

(d) $p=3$ and $n \geq \max (7, g+6,2 g+1)$.

MSC2000: $14 \mathrm{H} 30$.

Keywords: covers, moduli of curves, positive characteristic. 
In characteristic $p=0$, this has recently been proved by Magaard and Völklein [2004] (except for the case $(g, n)=(2,5)$ ). The cases $g=1$ and $p=0$ had been proved earlier by Fried, Klassen and Kopeliovich [Fried et al. 2001]. Also in characteristic 0, Artebani and Pirola [2003] have shown that every curve admits a tame rational function of degree $n$ with alternating monodromy group, provided that $n \geq 12 g+4$. In characteristic $p \neq 3$, Schröer [2003] has proved that for every $g \geq 0$ there exists some curve of genus $g$ admitting a rational function with monodromy group $A_{n}$, for certain values of $n$.

Note that there are classical analogs of these results for the symmetric group. In fact, one knows that every curve of genus $g \geq 2$ over an algebraically closed field of characteristic $p \neq 2$ admits a tame rational function of degree $n$ with monodromy group $S_{n}$, provided that $2 n-2 \geq g$ [Fulton 1969]. However, in characteristic 2, it seems to be unknown whether every curve admits a tame rational function at all no matter what the degree and the monodromy group are.

Our results are somewhat more precise than Theorem 1 above: the tame cover $f: X \rightarrow \mathbb{P}_{k}^{1}$ can be chosen in such a way that its inertia groups are all generated by 3 -cycles (except if $p=3$ or if $p=2$ and $n+g$ is odd). If $p \neq 2$ and $n \geq$ $\max (g+6,2 g+1)$, the cover $f$ can be chosen in such a way that its inertia groups are all generated by double transpositions (with the possible exception $(p, g, n)=$ $(3,0,6))$. See the statement of Theorem 10.

For $p \neq 2,3$ our results are optimal, in the following sense. If $n<g+3$ or $n<2 g+1$ then the generic curve of genus $g$ does not admit a tame rational function of degree $n$ with alternating monodromy group. (For $g \geq 3$, this is shown in [Magaard and Völklein 2004]; see the proof of Theorem 3.3 there. For $g<3$, one can use similar arguments.)

For $p=2$ or $p=3$, it is unlikely that our results are optimal. For instance, we believe (but were not able to show) that the generic curve of genus 1 in characteristic 2 admits a tame rational function of degree 5 with alternating monodromy. If this were the case, the situation in characteristic 2 would be the same as in characteristic 0 ; that is, the condition that $n+g$ is odd in Theorem 1(b) would be unnecessary (except for the case $(g, n)=(0,4)$, which does not occur in characteristic 2$)$. This would then also be an optimal result. But at the moment, there are infinitely many pairs $(g, n)$ which, to our knowledge, may or may not occur in characteristic 2 . In characteristic 3 , there are only finitely many such cases. See Section 3.3 for a list of all open cases.

1.2. To show the existence of rational functions with alternating monodromy, Magaard and Völklein [2004] use Hurwitz spaces, i.e., moduli space for covers of $\mathbb{P}^{1}$. The main result of their paper can be stated as follows: the natural map $\mathcal{H}_{r, n} \rightarrow M_{g}$ from a certain Hurwitz space to the moduli space of curves (which 
maps the isomorphism class of a cover $f: X \rightarrow \mathbb{P}^{1}$ to the isomorphism class of the curve $X$ ) has a dense image. In [Fried and Völklein 1991], Hurwitz spaces are constructed in characteristic 0 , using tools from topology and the theory of complex analytic functions. From this point of view, it seems difficult to extend the results of [Magaard and Völklein 2004] to positive characteristic. However, it is shown in [Fulton 1969] and [Wewers 1998] that Hurwitz spaces can also be constructed in a purely algebraic way and therefore make sense in positive characteristic, too. Moreover, for almost all primes $p$ a given Hurwitz space has good reduction from characteristic 0 to characteristic $p$-in the case of the Hurwitz space $\mathscr{H}_{r, n}$ used in [Magaard and Völklein 2004], this is true for all primes $p>n$. Using this good reduction result, it is then easy to extend the results from that paper to characteristic $p$, provided that $p>n$.

However, for small primes $p$ this kind of argument does not work. For instance, if $p \leq n$ the Hurwitz space $\mathscr{H}_{n, r}$ may have bad reduction to characteristic $p$. This makes it very difficult to study $\mathscr{H}_{n, r} \otimes \mathbb{F}_{p}$, using results on $\mathscr{H}_{n, r} \otimes \mathbb{Q}$. The same phenomenon prevented Fulton [1969] from proving the irreducibility of $\mathcal{M}_{g} \otimes \mathbb{F}_{p}$ for $p \leq 2 g+1$.

Luckily, it turns out that most of the arguments in [Magaard and Völklein 2004] (for instance, the induction step from $(g, n)$ to $(g+1, n+1))$ can be carried out in a purely algebraic way. In fact, Hurwitz spaces are not really essential; the key results that are used are the geometry of the boundary of $\mu_{g}$ and the deformation theory of tame covers, developed in [Grothendieck 1971]. Both these tools are algebraic and work in any characteristic. The situation is different for the proof of Theorem 1 for small values of $g$ and $n$, where the induction process starts. Here the arguments in [Magaard and Völklein 2004] are based on Riemann's Existence Theorem and do not carry over to characteristic $p$ if $p$ is small. This is the reason why the statement of Theorem 1 is weaker for $p=2,3$.

\section{The two induction steps}

2.1. Deformation of admissible covers. Let $k$ be an algebraically closed field. We choose a compatible system $\left(\zeta_{n}\right)$ of $n$-th roots of unity in $k$, where $n$ runs over all natural numbers prime to the characteristic of $k$. Let $t$ denote a transcendental element over $k$. Our goal is to construct tame covers $f_{\eta}: X_{\eta} \rightarrow \mathbb{P}_{k((t))}^{1}$ over the field $k((t))$ by deforming a given tame cover $f_{s}: X_{s} \rightarrow Z_{s}$ between singular curves. The standard general reference for the deformation theory of tame covers is of course [Grothendieck 1971]. For the particular results that we use, we refer to [Saïdi 1997], [Harbater and Stevenson 1999] or [Wewers 1999].

Let $G$ be a finite group. For $i=1,2$, we have a subgroup $G_{i} \subset G$, a tame $G_{i}$-Galois cover $h_{i}: Y_{i} \rightarrow \mathbb{P}_{k}^{1}$ between smooth projective curves over $k$, and a 
closed point $y_{i} \in Y_{i}$. We assume that the datum $\left(G, G_{i}, h_{i}, y_{i}\right)$ has the following properties. First, we assume that $G$ is generated by its subgroups $G_{1}$ and $G_{2}$. For the second condition, let $g_{i} \in G_{i}$ be the canonical generator of the stabilizer of $y_{i}$, with respect to $\left(\zeta_{n}\right)$. (An element $g \in G_{i}$ with $g\left(y_{i}\right)=y_{i}$ is called a canonical generator if there exists a formal parameter $u$ at $y_{i}$ such that $g^{*} u=\zeta_{n} \cdot u$, with $n=\operatorname{ord}(g)$.) Then we demand that $g_{1}=g_{2}^{-1}$. We denote by $n_{0}$ the order of $g_{1}=g_{2}^{-1}$.

Given $\left(G, G_{i}, h_{i}, y_{i}\right)$ satisfying the preceding conditions, we construct a tame $G$-Galois cover $h_{s}: Y_{s} \rightarrow Z_{s}$ between semistable curves over $k$, as follows. We set

$$
Y_{s}:=\left(\operatorname{Ind}_{G_{1}}^{G}\left(Y_{1}\right) \amalg \operatorname{Ind}_{G_{2}}^{G}\left(Y_{2}\right)\right) / \sim,
$$

where $\sim$ denotes the following equivalence relation. A point $y \in \operatorname{Ind}_{G_{1}}^{G}\left(Y_{1}\right)$ is equivalent to a point $y^{\prime} \in \operatorname{Ind}_{G_{2}}^{G}\left(Y_{2}\right)$ if and only if there exists an element $g \in G$ with $y=g\left(y_{1}\right)$ and $y^{\prime}=g\left(y_{2}\right)$. It is easy to see that the set $Y_{s}$ is naturally equipped with the structure of a semistable curve over $k$ and with a $k$-linear action of $G$. The curves $Y_{1} \subset Y_{s}$ and $Y_{2} \subset Y_{s}$ are irreducible components of $Y_{s}$, with stabilizers $G_{1}$ and $G_{2}$. Moreover, the points $y_{1} \in Y_{1}$ and $y_{2} \in Y_{2}$ correspond to the same (singular) point of $Y_{s}$. We define $Z_{s}:=Y_{s} / G$ as the quotient scheme. The scheme $Z_{s}$ is a semistable curve over $k$, with two irreducible components $Z_{1}, Z_{2}$ which meet in one points. The components $Z_{i}$ can be identified with $\mathbb{P}_{k}^{1}$, via the covers $h_{i}$. The natural map $h_{s}: Y_{s} \rightarrow Z_{s}$ is a tame admissible cover; see [Wewers 1999].

It is easy to construct a semistable curve $\mathscr{E}$ over Spec $k \llbracket t \rrbracket$ with special fiber $Z_{s}$ and with generic fiber $Z_{\eta}=\mathbb{P}_{k((t))}^{1}$, satisfying the following additional property: the complete local ring of $\mathscr{E}$ at the singular point of the special fiber is isomorphic to $k \llbracket t, u, v \mid u v=t^{n_{0}} \rrbracket$. Let $\bar{\tau}_{1}, \ldots, \bar{\tau}_{s} \in Z_{1}=\mathbb{P}_{k}^{1}$ denote the branch points of $h_{1}$ distinct from $h_{1}\left(y_{1}\right)$, and $\bar{\tau}_{s+1}, \ldots, \bar{\tau}_{r}$ the branch points of $h_{2}$ distinct from $h_{2}\left(y_{2}\right)$. Lift these points to $k \llbracket t \rrbracket$-rational points $\tau_{1}, \ldots \tau_{r}$ of $\mathscr{L}$. By [Wewers 1999, Theorem 3.1.1], there exists a tame $G$-Galois cover $h:$ y $\rightarrow \mathscr{L}$ between semistable curves over $k \llbracket t \rrbracket$, étale over $\mathscr{E}-\left\{\tau_{1}, \ldots, \tau_{r}\right\}$ whose special fiber is equal to the cover $h_{s}$. The generic fiber $h_{\eta}: Y_{\eta} \rightarrow \mathbb{P}_{k((t))}^{1}$ is a tame $G$-Galois cover between smooth projective curves, with $r$ branch points. We say that $h_{\eta}$ is a smooth Galois cover associated to $\left(G, G_{i}, h_{i}, y_{i}\right)$.

Suppose that $G \subset S_{n}$ is a transitive permutation group on $n$ letters, and let $H \subset G$ denote the stabilizer of 1 in $G$. Let $\mathscr{X}:=\mathscr{y} / H$ denote the quotient scheme. Then $\mathscr{X}$ is a semistable curve over $k \llbracket t \rrbracket$, with generic fiber $X_{\eta}=Y_{\eta} / H$ and special fiber $X_{s}=Y_{s} / H$ (for the second equality we have used the fact that the cover $h_{s}: Y_{s} \rightarrow Z_{s}$ is separable). Moreover, $h_{\eta}$ is the Galois closure of the cover $f_{\eta}: \mathscr{X}_{\eta} \rightarrow \mathbb{P}_{k((t))}^{1}$. Therefore, the cover $f_{\eta}$ is tame, with monodromy group $G$. We say that $f_{\eta}$ is a smooth cover associated to $\left(G \subset S_{n}, G_{i}, h_{i}, y_{i}\right)$. 
It is easy to describe the special fiber $X_{s}$ and the admissible cover $f_{s}: X_{s} \rightarrow Z_{s}$ in terms of the datum $\left(G \subset S_{n}, G_{i}, h_{i}, y_{i}\right)$. For instance, the irreducible components of $X_{s}$ lying above the component $Z_{i} \subset Z_{s}$ are in bijection with the orbits of the $G_{i}$-action on $\{1, \ldots, n\}$. For each orbit $O \subset\{1, \ldots, n\}$, the restriction of $f_{s}$ to the component $X_{O} \subset X_{s}$ is isomorphic to the quotient cover of $h_{i}: Y_{i} \rightarrow Z_{i}=\mathbb{P}_{k}^{1}$ corresponding to the stabilizer of some element of $O$.

2.2. A useful lemma. Let $X$ be a generic curve of genus $g \geq 1$, defined over an algebraically closed field $k$ of characteristic $p \geq 0$. Let $f: X \rightarrow \mathbb{P}_{k}^{1}$ be a tame cover, with monodromy group $G$ and with branch points $t_{1}, \ldots, t_{r}, r \geq 3$. Without loss of generality, we may assume that $t_{1}=0, t_{2}=1$ and $t_{3}=\infty$. We say that $f$ has generic branch points if the branch points $t_{4}, \ldots, t_{r}$, considered as elements of $k$, are algebraically independent over the prime field $k_{0} \subset k$.

The following lemma will be useful in the proof of Theorem 1.

Lemma 2. Let $k^{\prime} / k$ be a field extension, $z: \operatorname{Spec} k^{\prime} \rightarrow \mathbb{P}_{k}^{1}$ a generic point and $x_{1}, x_{2}:$ Spec $k^{\prime} \rightarrow X$ two distinct $k^{\prime}$-rational points with $z=f\left(x_{1}\right)=f\left(x_{2}\right)$. Suppose that one of the following conditions holds.

(a) We have $g=1$ and the monodromy group $G$ is doubly transitive.

(b) We have $g \geq 2$ and $f$ has $r>3 g$ generic branch points.

Then $\left(X \otimes k^{\prime} ; x_{1}, x_{2}\right)$ is a generic two-pointed curve, meaning that its classifying map Spec $k^{\prime} \rightarrow M_{g, 2}$ has a Zariski dense image.

Proof. Assume that condition (a) holds. Denote by $\varphi_{1}:$ Spec $k^{\prime} \rightarrow M_{1,1}$ the classifying map of the one-pointed curve $\left(X \otimes k^{\prime}, x_{1}\right)$ and by $\varphi_{2}:$ Spec $k^{\prime} \rightarrow \mathcal{M}_{1,2}$ the classifying may of the two-pointed curve $\left(X \otimes k^{\prime} ; x_{1}, x_{2}\right)$. Recall that $\mu_{1,1} \cong \mathbb{A}^{1}$ and that $\varphi_{1}$ is simply the $j$-invariant of the elliptic curve $\left(X \otimes k^{\prime}, x_{1}\right)$. It is well known that the $j$-invariant of an elliptic curve depends only on the underlying curve and not on the distinguished point (i.e. $M_{1,1} \cong M_{1}$ ). Hence we may regard $\varphi_{1}$ as an element of $k$. Since $X$ is generic, $\varphi_{1}$ is transcendental over $k_{0}$. It follows that the image of $\varphi_{2}$ is contained in the fiber $M_{1,2} \otimes k_{0}\left(\varphi_{1}\right)$ of the natural map $M_{1,2} \rightarrow M_{1,1}$ over Spec $k_{0}\left(\varphi_{1}\right)$. To prove the lemma, it suffices to show that the image of $\varphi_{2}$ is Zariski dense in $\mathcal{M}_{1,2} \otimes k_{0}\left(\varphi_{1}\right)$. Since $\mathcal{M}_{1,2} \otimes k_{0}\left(\varphi_{1}\right)$ is 1-dimensional, it even suffices to find a $k$-rational place $v$ of $k^{\prime}$ such that the image of $v$ under $\varphi_{2}$ lies on the boundary of $\mu_{1,2} \otimes k_{0}\left(\varphi_{1}\right)$. In other words, we require $x_{1}(v)=x_{2}(v)$ (here $x_{i}(v)$ denotes the $k$-rational point on $X$ obtained by "specializing" $x_{i}$ at $v$ ).

Let $S \subset X \times_{k} X$ denote the locus of pairs $(x, y)$ with $f(x)=f(y)$. It is a 1dimensional closed subset, and the natural map $S \rightarrow \mathbb{P}_{k}^{1}$ is finite. The pair $\left(x_{1}, x_{2}\right)$ is a $k^{\prime}$-rational point on $S$ which maps to the generic point of $\mathbb{P}_{k}^{1}$. Therefore, the closure of the image of $\left(x_{1}, x_{2}\right)$ is an irreducible component $S^{\prime}$ of $S$, distinct from the diagonal $\Delta \subset X \times_{k} X$. We have to show that $S^{\prime}$ has nonempty intersection with 
$\Delta$. The assumption that $G$ is doubly transitive implies that $S-\Delta$ is irreducible. Hence we have $S^{\prime}=\overline{S-\Delta}$. On the other hand, since the cover $f: X \rightarrow \mathbb{P}_{k}^{1}$ is not étale, $\Delta$ has nonempty intersection with $S^{\prime}=\overline{S-\Delta}$. This proves Lemma 2, assuming condition (a).

Assume now that condition (b) holds. Let $k_{1} \subset k$ denote the minimal algebraically closed subfield of $k$ over which the curve $X$ can be defined. Since $X$ is generic, $k_{1}$ is isomorphic to the algebraic closure of the function field of $\mu_{g}$. In particular, tr.deg $\left(k_{1} / k_{0}\right)=3 g-3$. Write $X=X_{1} \otimes_{k_{1}} k$.

By assumption, the branch points $t_{4}, \ldots, t_{r}$ of $f$, considered as elements of $k$, are algebraically independent over $k_{0}$. Moreover, we have $r-3>3 g-3=$ $\operatorname{tr} \cdot \operatorname{deg}\left(k_{1} / k_{0}\right)$. It follows that the cover $f: X_{1} \otimes k \rightarrow \mathbb{P}_{k}^{1}$ is not isotrivial, with respect to the extension $k / k_{1}$. More precisely, the subfield $k(f) \subset k(X)$ cannot be generated over $k$ by an element $f_{1} \in k_{1}\left(X_{1}\right)$.

Let $f_{V}: X_{1} \times V \rightarrow \mathbb{P}_{V}^{1}$ be a model of $f$ over $V$, where $V$ is a variety over $k_{1}$ (this means that $f$ is the pullback of $f_{V}$ via a generic point Spec $k \rightarrow V$ ). For each closed point $v \in V$ we obtain, by specializing $f_{V}$, a tame cover $f_{v}: X_{1} \rightarrow \mathbb{P}_{k_{1}}^{1}$. Since $f$ is not isotrivial, there exist infinitely many points $v \in V$ which give rise to pairwise weakly nonisomorphic covers $f_{v}$. Now [Magaard and Völklein 2004, Lemma 2.3] shows that the locus $S \subset X_{1} \times_{k_{1}} X_{1}$ of pairs of points $(x, y)$ which satisfy $f_{v}(x)=f_{v}(y)$ for all $v \in V$ is Zariski dense in $X_{1} \times_{k_{1}} X_{1}$. On the other hand, since $S \rightarrow \mathbb{P}_{k_{1}}^{1}$ is finite and $f\left(x_{1}\right)=f\left(x_{2}\right)=z$ is generic, the pair $\left(x_{1}, x_{2}\right)$ is a generic point of $S$. This proves Lemma 2, assuming condition (b).

Remark 3. Lemma 2 is very similar to [Magaard and Völklein 2004, Lemma 2.4], at least in the case $g \geq 2$. In the case $g=1$, our lemma improves on theirs by removing the assumption that $f$ depends on more parameters than $X$. This is used in the proof of Theorem 1 for the case $(g, n)=(2,5)$.

2.3. The first induction step. As before, we let $X$ be a generic curve of genus $g \geq 0$, defined over an algebraically closed field $k$ of characteristic $p \geq 0$. The following proposition will serve as an induction step in the proof of Theorem 1, from $(g, n)$ to $(g, n+2)$.

Proposition 4. Assume that there exists a tame cover $f: X \rightarrow \mathbb{P}_{k}^{1}$ of degree $n \geq 3$, with monodromy group $A_{n}$ and with $r$ branch points. Let $k^{\prime}:=k((t))^{\mathrm{alg}}$ and $X^{\prime}:=X \otimes_{k} k^{\prime}$. Then there exists a tame cover $f^{\prime}: X^{\prime} \rightarrow \mathbb{P}_{k^{\prime}}^{1}$ of degree $n+2$, with monodromy group $A_{n+2}$ and with $r+2$ branch points.

Proof. Let $h_{1}: Y_{1} \rightarrow \mathbb{P}_{k}^{1}$ be the Galois closure of $f$. By assumption, the Galois group of $h_{1}$ is $G_{1}:=A_{n}$, which we view as a subgroup of $G:=A_{n+2}$, in the obvious way. Let $y_{1} \in Y_{1}$ be any closed point where $h_{1}$ is unramified. We let $h_{2}: Y_{2} \rightarrow \mathbb{P}_{k}^{1}$ be a cyclic cover of order 3 or 2 (according to whether $p \neq 3$ 
or $p=3$ ), ramified at two points. We identify the Galois group of $h_{2}$ with the subgroup $G_{2} \subset G$ generated by the 3 -cycle $(n, n+1, n+2)$ in the case $p \neq 3$ or by the double transposition $(n-1, n+1)(n, n+2)$ in the case $p=3$. In either case, $G=\left\langle G_{1}, G_{2}\right\rangle$. We also choose a point $y_{2} \in Y_{2}$ where $h_{2}$ is unramified. By the construction of Section 2.1, there exists a tame $G$-Galois cover $h^{\prime}: Y^{\prime} \rightarrow \mathbb{P}_{k^{\prime}}^{1}$ which lifts the datum $\left(G, G_{i}, h_{i}, y_{i}\right)$. Let $H \subset G$ be the stabilizer of 1 . Set $X^{\prime}:=Y^{\prime} / H$ and let $f^{\prime}: X^{\prime} \rightarrow \mathbb{P}_{k^{\prime}}^{1}$ denote the natural map. By construction, $f^{\prime}$ is a tame cover of degree $n+2$, with monodromy group $A_{n+2}$ and with $r+2$ branch points.

It remains to prove that $X^{\prime} \cong X \otimes_{k} k^{\prime}$. The Riemann-Hurwitz formula shows that the genus of $X^{\prime}$ is equal to $g$. Moreover, by construction, one of the components of the stable reduction of $X^{\prime}$ is isomorphic to $X$. It follows that $X^{\prime}$ has good reduction and specializes to a generic curve of genus $g$. Therefore, $X^{\prime}$ is itself generic, hence $X^{\prime} \cong X \otimes_{k} k^{\prime}$.

2.4. The second induction step. For the proof of Theorem 1, we need another induction step, going from $(g, n)$ to $(g+1, n+1)$. As in the last subsection, $X$ is a generic curve of genus $g \geq 0$ over $k$.

Proposition 5. Assume that there exists a tame cover $f: X \rightarrow \mathbb{P}_{k}^{1}$ of degree $n \geq 3$, with monodromy group $A_{n}$ and with $r$ branch points. Assume, moreover, that either

(i) $g \leq 1$, or

(ii) $g \geq 2$ and $r>3 g$.

Then there exists a field extension $k^{\prime} / k$, a generic curve $X^{\prime}$ of genus $g+1$ over $k^{\prime}$ and a tame cover $f^{\prime}: X^{\prime} \rightarrow \mathbb{P}_{k^{\prime}}^{1}$ of degree $n+1$, with monodromy group $A_{n+1}$ and with $r+2$ branch points.

Proof. We are allowed to replace the field $k$ by an arbitrary extension $k^{\prime}$. We may therefore assume that the cover $f: X \rightarrow \mathbb{P}_{k}^{1}$ has generic branch points, see Section 2.2. (To see this, let $\mathscr{H}_{n, r}$ denote the Hurwitz space parameterizing tame covers of $\mathbb{P}^{1}$ of degree $n$ with $r$ branch points. By [Wewers 1998, Proposition 4.2.1], $\mathscr{H}_{n, r}$ is a smooth scheme of relative dimension $r$ over $\mathbb{Z}$. The cover $f$ of the proposition corresponds to a $k$-rational point on $\mathscr{H}_{n, r} \otimes k_{0}$. We may thus replace $f$ by the generic cover corresponding to the connected component of $\mathcal{H}_{n, r} \otimes k_{0}^{\text {alg }}$ on which this point lies.)

Let $z: \operatorname{Spec} k_{1} \rightarrow \mathbb{P}_{k}^{1}$ be a generic geometric point. Let $h_{1}: Y_{1} \rightarrow \mathbb{P}_{k_{1}}^{1}$ denote the Galois closure of $f \otimes k_{1}$. We identify the Galois group of $h_{1}$ with $G_{1}:=A_{n}$, which we regard as a subgroup of $G:=A_{n+1}$, in the obvious way. We choose a point $y_{1} \in Y_{1}$ which lies above $z \in \mathbb{P}_{k_{1}}^{1}$. If $p \neq 3$ (resp. if $p=3$ ), we let $h_{2}: Y_{2} \rightarrow \mathbb{P}_{k_{1}}^{1}$ be a cyclic cover of order 3 (resp. of order 2), ramified at two points. We identify the Galois group of $h_{2}$ with the subgroup $G_{2} \subset G$ generated by the 3 -cycle $(n-1, n, n+1)$ (resp. by the double transposition $(1,2)(n, n+1))$. We 
also choose a point $y_{2} \in Y_{2}$ where $h_{2}$ is unramified. Let $k^{\prime}:=k_{1}((t))^{\text {alg }}$. We lift the datum $\left(G, G_{i}, h_{i}, y_{i}\right)$ to a tame $G$-Galois cover $h^{\prime}: Y^{\prime} \rightarrow \mathbb{P}_{k^{\prime}}^{1}$, see Section 2.1. Let $H \subset G$ be the stabilizer of 1 . Set $X^{\prime}:=Y^{\prime} / H$ and let $f^{\prime}: X^{\prime} \rightarrow \mathbb{P}_{k^{\prime}}^{1}$ denote the natural map. By construction, $f^{\prime}$ is a tame cover of degree $n+1$, with monodromy group $A_{n+1}$ and with $r+2$ branch points. The Riemann-Hurwitz formula shows that $X^{\prime}$ has genus $g+1$. It remains to prove that $X^{\prime}$ is generic.

Let $\mathscr{X}$ be the semistable model of $X^{\prime}$ over $k_{1} \llbracket t \rrbracket$ originating from the construction of Section 2.1. If $p \neq 3$, then the special fiber $X_{s}$ of $\mathscr{X}$ contains the curves $X \otimes k_{1}$ and $Y_{2}$ as irreducible components. These components meet in two points $x_{1}, x_{2} \in X$ with $f\left(x_{1}\right)=f\left(x_{2}\right)=z$. The other components of $X_{s}$ are copies of $\mathbb{P}_{k_{1}}^{1}$. If $p=3$, the situation is similar. The only difference is that $X_{s}$ contains two copies of $Y_{2}$ as irreducible components, corresponding to the orbits of length two of $(1,2)(n, n+1)$ acting on $\{1,2, \ldots, N\}$.

If $g=0$, the above description of $X_{s}$ shows that $X^{\prime}$ has bad reduction and is therefore a generic curve of genus 1 . Hence we may assume that $g \geq 1$. Let $\mathscr{X}^{\text {stab }}$ be the stable model of $X^{\prime}$ over $k_{1} \llbracket t \rrbracket$ (i.e. the minimal semistable model). The curve $\mathscr{X}^{\text {stab }}$ is obtained from the curve $\mathscr{X}$ by blowing down all components of $X_{S}$ of genus 0 which contain less than three singular points. It follows that the special fiber of $\mathscr{Q}^{\text {stab }}$ is isomorphic to the curve $X \otimes k_{1}$, with the points $x_{1}$ and $x_{2}$ identified. Let

$$
\varphi: \text { Spec } k_{1} \llbracket t \rrbracket \rightarrow \bar{M}_{g+1}
$$

denote the classifying map for $\mathscr{X}^{\text {stab }}$, and write $\varphi_{\eta}$ (resp. $\varphi_{S}$ ) for the restriction of $\varphi$ to the generic (resp. the closed) point of Spec $k_{1} \llbracket t \rrbracket$. We have to show that the image of $\varphi_{\eta}$ is Zariski dense in $M_{g+1}$. By our description of the special fiber, the image of $\varphi_{s}$ lies on a boundary component $T$ of $\bar{M}_{g+1}$ which is isomorphic to $\mu_{g, 2}$. Moreover, $\varphi_{s}$ corresponds, under this isomorphism, to the 2-pointed curve $\left(X \otimes k_{1} ; x_{1}, x_{2}\right)$ [Knudsen 1983]. It follows from Lemma 2 that the image of $\varphi_{s}$ is Zariski dense in the boundary component $T$ (note that $A_{n}$ is doubly transitive for $n \geq 4$ and that the case $n=3$ does not occur for $g \geq 1$ ). But $T$ has codimension one in $\bar{M}_{g+1}$ [Deligne and Mumford 1969]. Therefore, the image of $\varphi_{\eta}$ is Zariski dense in $M_{g+1}$. This concludes the proof of Proposition 5 .

\section{The main result}

3.1. The case $g=0$. Proposition 7 below implies Theorem 1(a). In its proof we use the following easy lemma.

Lemma 6. Let $p \neq 2$. Choose $\lambda \in k-\{0,1\}$ such that

$$
\Phi(\lambda)=\sum_{i=0}^{(p-1) / 2}\left(\begin{array}{c}
(p-1) / 2 \\
i
\end{array}\right)^{2} \lambda^{i}
$$


is nonzero. Then there exists a cover $f: \mathbb{P}_{k}^{1} \rightarrow \mathbb{P}_{k}^{1}$ of degree $p$ branched at $0,1, \lambda, \infty$ with monodromy group $D_{p}$. For $x=0,1, \lambda, \infty$, the inverse image of $x$ consists of $(p-1) / 2$ ramification points of order two and one unramified point.

Proof. Choose $\lambda \in k-\{0,1\}$ such that $\Phi(\lambda)$ is nonzero. This implies that the elliptic curve $E$ defined by $y^{2}=x(x-1)(x-\lambda)$ is ordinary; see [Hartshorne 1977, Corollary IV.4.22], for example. Let $E^{\prime} \rightarrow E$ be an étale isogeny of degree $p$. Taking the quotient on both sides under the elliptic involution, we obtain a rational map $f: \mathbb{P}_{k}^{1} \rightarrow \mathbb{P}_{k}^{1}$ of degree $p$ as in the statement of the lemma.

Proposition 7. Let $n \geq 3$ and $p \geq 0$. Suppose that $n \neq 4$ if $p=2$ and $n \geq 7$ if $p=3$. Then there exists a tame cover $f: \mathbb{P}_{k}^{1} \rightarrow \mathbb{P}_{k}^{1}$ of degree $n$ with monodromy group $A_{n}$, defined over an algebraically closed field $k$ of characteristic $p$. More precisely:

(a) If $p \neq 2,3$, we may choose $f$ with $r=n-1$ branch points and ramification type $(3, \ldots, 3)$.

(b) Suppose that one of the following holds:

(i) $p \geq 7$ and $n \geq 6$,

(ii) $p=3$ and $n \geq 7$, or

(iii) $p=5$ and $n \geq 7$ is odd.

Then we may choose $f$ with $r=n-1$ branch points and ramification type $(2-2, \ldots, 2-2)$.

(c) If $p=2$ and $n$ is odd, we may choose $f$ with $r=n-1$ branch points and ramification type $(3, \ldots, 3)$.

(d) If $p=2$ and $n \geq 6$ is even, we may choose $f$ with $r=n-3$ branch points, and ramification type $(5,3-3,3, \ldots, 3)$.

Proof. Suppose first that $p \neq 2,3$ and consider the case of triple ramification. The case $n=3$ is trivial, as we may take for $f$ the function $f(x)=x^{3}$. For $n=4$, we set

$$
f(x):=\frac{x^{3}(x-2)}{1-2 x}=\frac{(x-1)^{3}(x+1)}{1-2 x}+1 .
$$

It is obvious that $f$ has ramification locus $\{0,1, \infty\}$ and ramification index 3 in each of these points. (Note that we use $p \neq 2,3$.) The monodromy group of $f$ is a transitive permutation group on 4 letters generated by 3-cycles, hence isomorphic to $A_{4}$. This settles the case $n=4$. The cases $n>4$ follow from $n=3,4$ by induction, using Proposition 4. This proves (a).

We now prove (b). If $p \geq 7$ and $n=6$, we may use Riemann's Existence Theorem, since in this case $p$ does not divide the order of $A_{6}$. For example, one 
checks using GAP that the tuple

$$
((1,2)(3,4) ;(3,6)(4,5) ;(2,6)(3,4) ;(2,6)(3,5) ;(1,2)(4,6))
$$

generates $A_{6}$.

Next we handle the case $p \neq 2$ and $n=7$. Let $f_{1}: \mathbb{P}_{k}^{1} \rightarrow \mathbb{P}_{k}^{1}$ be a separable map of degree four branched at four points $0,1, \lambda, \infty$ with ramification type $(2,2,2,2)$ and monodromy group $G_{1}$ the dihedral group of order 8 . For $p \neq 2$, the existence of such a cover follows from Riemann's Existence Theorem. Write $h_{1}: Y_{1} \rightarrow$ $\mathbb{P}_{k}^{1}$ for the Galois closure of $f_{1}$ and choose a point $y_{1} \in Y_{1}$ above $x=0 \in \mathbb{P}_{k}^{1}$. Choose an embedding of $G_{1}$ into $A_{7}$ such that $G_{1}$ acting on $\{1,2, \ldots, 7\}$ has orbits $\{1,2\},\{3,4,6,7\},\{5\}$. Write $\left(g_{1}, g_{2}, g_{3}, g_{4}\right)$ for the corresponding tuple of double transpositions. Choose $\mu \in k-\{0,1\}$. If $p=5$ we suppose moreover that $\mu$ is not a primitive 3rd root of unity. Let $f_{2}: \mathbb{P}_{k}^{1} \rightarrow \mathbb{P}_{k}^{1}$ be a separable map of degree five branched at $0,1, \mu, \infty$ with monodromy group the dihedral group of order 10 and with ramification type (2-2, 2-2, 2-2, 2-2). If $p \neq 5$ the existence of $f_{2}$ follows from Riemann's Existence Theorem. If $p=5$ it follows from Lemma 6. Write $h_{2}$ : $Y_{2} \rightarrow \mathbb{P}_{k}^{1}$ for the Galois closure of $f_{2}$ and choose a point $y_{2} \in Y_{2}$ above $x=0 \in \mathbb{P}_{k}^{1}$. Let $G_{2}$ denote the Galois group of $h_{2}$. We embed $G_{2}$ into $A_{7}$ such that the action of $G_{2}$ on $\{1,2, \ldots, 7\}$ has orbits $\{1,2,3,4,5\},\{6\},\{7\}$. Write $\left(g_{1}^{\prime}, g_{2}^{\prime}, g_{3}^{\prime}, g_{4}^{\prime}\right)$ for the tuple of transpositions corresponding to the cover $f_{2}$. We may arrange things such that $g_{1}=g_{1}^{\prime}$. One checks that $A_{7}$ is generated by $G_{1}$ and $G_{2}$. Therefore the results of Section 2.1 imply that there exists a map $\mathbb{P}_{k}^{1} \rightarrow \mathbb{P}_{k}^{1}$ of degree $n=7$ with monodromy group $A_{7}$ and ramification (2-2, 2-2, 2-2, 2-2, 2-2, 2-2). This settles the case $p \neq 2$ and $n=7$.

Parts (i) and (iii) of (b) now follow from the cases $n=6,7$ by induction, using Proposition 4. In order to prove part (ii) of (b) we need an extra construction to handle the case $n=8$, because the preceding construction for $n=6$ worked only for $p \geq 7$.

We define (in characteristic 3 ) a tame cover $f_{1}: \mathbb{P}_{k}^{1} \rightarrow \mathbb{P}_{k}^{1}$ of degree 5 , ramification type $(2-2,2-2,5)$ and Galois group $D_{5}$. Since 3 does not divide the order of the Galois group, we can use Riemann's Existence Theorem. For example, we may choose $f_{1}$ so that it corresponds to the triple $((1,2)(3,4) ;(1,5)(2,3) ;(1,5,2,4,3))$.

We also define (in characteristic 3 ) a cover

$$
f_{2}(x)=\frac{x^{5}(x+1)}{x-1}=\frac{\left(x^{2}-x-1\right)^{2}\left(x^{2}+1\right)}{x-1}+1 .
$$

The cover $f_{2}: Y_{2} \rightarrow \mathbb{P}_{k}^{1}$ is branched at $x=0,1, \infty$, with ramification type (5, 2-2, 5). One easily checks that this is the only such cover in characteristic 3 , up to isomorphism. Let $G_{2} \subset A_{6}$ be its monodromy group. One checks, for example using GAP, that $G_{2}$ is either isomorphic to $\mathrm{PSL}_{2}(5) \cong A_{5}$ or to $A_{6}$. 
We claim that $G_{2} \simeq \mathrm{PSL}_{2}$ (5). (One does not actually need this in what follows.) It follows from [Serre 1992, Proposition 7.4.3] that there is a unique $\mathrm{PSL}_{2}$ (5)Galois cover $g: Y \rightarrow \mathbb{P}_{\overline{\mathbb{Q}}}^{1}$ branched at three points with ramification type $(5,2-2,5)$ over $\overline{\mathbb{Q}}$, up to isomorphism. In fact, this cover may be defined over $\mathbb{Q}$. It follows from the discussion in [Bouw and Pries 2003, §2.4] that $g$ has good reduction to characteristic $p=3$. Here one uses that $p$ exactly divides the order of $\mathrm{PSL}_{2}$ (5). Since $f_{2}$ is the unique cover (up to isomorphism) of degree 6 with ramification type $(5,2-2,5)$ in characteristic 3 , we conclude that the reduction of $g$ to characteristic 3 is isomorphic to $f_{2}$. This shows that $G_{2} \cong \mathrm{PSL}_{2}(5)$.

Let $g_{1}, g_{3} \in G_{2}$ be canonical generators of inertia at $x=0, \infty$, respectively, with respect to some fixed 5-th root of unity. Let $f_{3}=f_{1}$. For $i=1,3$, we choose an embedding of the monodromy group $G_{i}$ of $f_{i}$ into $\mathrm{PSL}_{2}(5)$ by identifying $G_{i}$ with the normalizer in $\mathrm{PSL}_{2}(5)$ of the subgroup generated by $g_{i}$. We may choose this identification such that the canonical generator of inertia of the ramification point of order 5 of $f_{i}$ is $g_{i}^{-1}$. Using the results of Section 2.1, we may patch the covers $f_{1}, f_{2}, f_{3}$, yielding a cover $f: \mathbb{P}_{k}^{1} \rightarrow \mathbb{P}_{k}^{1}$ of degree 6 with monodromy group $G_{2} \subset S_{6}$ and ramification type (2-2, 2-2, 2-2, 2-2, 2-2).

We now apply the construction of Proposition 4 to $f$. Note that it does not matter for the construction that the monodromy group of $f$ is $\mathrm{PSL}_{2}(5)$ and not $A_{6}$. This yields a cover $f^{\prime}: \mathbb{P}_{k}^{1} \rightarrow \mathbb{P}_{k}^{1}$ of degree 8 branched at 7 points of type $(2-2, \ldots, 2-2)$. Note that the monodromy group $G^{\prime}$ of $f^{\prime}$ is a transitive group on 8 letters which contains $\mathrm{PSL}_{2}(5)$ and is contained in $A_{8}$. One checks, e.g. using GAP, that the only such group is $A_{8}$ itself. This proves (b) for $p=3$ and $n=8$. Now part (ii) of (b) follows from the cases $n=7,8$ by induction, using Proposition 4. This completes the proof of (b).

We now prove (c) and (d). We suppose that $p=2$. The case $n=3$ is again trivial; the general case of (c) follows by induction, using Proposition 4. To prove (d), we have to start the induction with $n=6$. We define (in characteristic 2)

$$
f(x)=\frac{x^{3}\left(x^{3}+x^{2}+1\right)}{x+1}=\frac{\left(x^{2}+x+1\right)^{3}}{x+1}+1 .
$$

One checks that $f$ is a tame cover of degree 6 , branched at $\{0,1, \infty\}$ and with ramification type (3, 3-3, 5). A standard result in group theory (see [Huppert 1967, p. 171], for instance) shows that the monodromy group of $f$ is isomorphic to $A_{6}$. This settles the case $(p, n)=(2,6)$. Part (d) of the proposition follows now from the cases $n=6$ by induction, using Proposition 4 . This completes the proof of Proposition 7.

The statement of Proposition 7 is probably not optimal. In the next proposition we list all the cases where we can positively exclude the existence of a tame rational function on $\mathbb{P}^{1}$ with alternating monodromy. 
Proposition 8. (i) Let $f: \mathbb{P}_{k}^{1} \rightarrow \mathbb{P}_{k}^{1}$ be a tame rational function of degree $n=3$ or $n=4$, with monodromy group $A_{n}$. Then the ramification type of $f$ is $(3, \ldots, 3)$ (with $n-1$ branch points).

(ii) Suppose that $p=2$. Then there does not exist a tame rational function $f$ : $\mathbb{P}_{k}^{1} \rightarrow \mathbb{P}_{k}^{1}$ of degree 4 and with monodromy group $A_{4}$.

(iii) Suppose that $p=3$. Then there does not exist a tame rational function $f$ : $\mathbb{P}_{k}^{1} \rightarrow \mathbb{P}_{k}^{1}$ of degree $n \leq 5$ with monodromy group $A_{n}$.

Proof. The proof of (i) is trivial. To prove (ii), suppose that there does exist a tame cover $f: \mathbb{P}_{k}^{1} \rightarrow \mathbb{P}_{k}^{1}$ of degree 4 with monodromy group $A_{4}$. By (i), such a cover would be of type $(3,3,3)$. Such a cover would also lift to characteristic 0 , in a unique way. It is easy to check, using the rigidity criterion of [Serre 1992, Section 7.3], that there is a unique $A_{4}$-cover of $\mathbb{P}^{1}$ with ramification type $(3,3,3)$ in characteristic zero. Therefore, the cover we are looking for would be the reduction of the cover $(*)$ of page 193 to characteristic 2 . However, it is easy to check that the cover $(*)$ has bad reduction to characteristic 2 . This gives the desired contradiction and proves (ii).

To prove (iii), suppose that $p=3$. The cases $n=3,4$ are already excluded by (i). It is easy to see, using the Riemann-Hurwitz formula, that a tame cover $f: \mathbb{P}_{k}^{1} \rightarrow \mathbb{P}_{k}^{1}$ of degree 5 has ramification type $(5,5),(5,2-2,2-2)$ or $(2-2,2-2,2-2,2-2)$. In the first case the monodromy group is cyclic of order 5 , in the second case a dihedral group of order 10. So it remains to rule out the third case. Let $\left(g_{1}, g_{2}, g_{3}, g_{4}\right)$ be a 4-tuple of double transpositions in $A_{5}$ with $g_{1} g_{2} g_{3} g_{4}=1$. We claim that the elements $g_{1}, \ldots, g_{4}$ do not genererate $A_{5}$. To prove the claim, let $h:=g_{1} g_{2}$ and $G$ the group generated by $g_{1}, \ldots, g_{4}$. If $h=1$ then $G$ is generated by two elements of order 2 ; it is then isomorphic to $\mathbb{Z} / 2, S_{3}$ or $D_{5}$, but not to $A_{5}$. If $h$ is a 3 -cycle, then the subgroups

$$
G_{1}:=\left\langle g_{1}, g_{2}\right\rangle \subset A_{5} \quad \text { and } \quad G_{2}:=\left\langle g_{3}, g_{4}\right\rangle
$$

are both isomorphic to $S_{3}$ and contain the same subgroup of order 3. It follows easily that $G_{1}=G_{2}$ and hence that $G \cong S_{3}$. Finally, if $h$ is a 5-cycle, then $G_{1}$ and $G_{2}$ are dihedral groups of order 10 and contain the same cyclic group of order 5 . As before, it follows that $G=G_{1}=G_{2} \cong D_{5}$. This proves the claim and hence also Proposition 8.

Remark 9. The following cases are left open by Propositions 7 and 8:

(i) $p=2, n \geq 6$ even, and ramification type $(3, \ldots, 3)$,

(ii) $p=3, n=6$,

(iii) $p=5, n \geq 6$ even, and ramification type $(2-2, \ldots, 2-2)$. 
3.2. We next give a more precise version of Theorem 1 of the introduction.

Theorem 10. Let $g \geq 0$ and $n \geq 3$. Let $X$ be a generic curve of genus $g$, defined over an algebraically closed field $k$ of characteristic $p \geq 0$. Then there exists a tame rational function $f: X \rightarrow \mathbb{P}_{k}^{1}$ of degree $n$ with monodromy group $A_{n}$ in each of the following cases.

(a) If $p \neq 2,3$ and $n \geq \max (g+3,2 g+1)$, we may choose $f$ with $r=g+n-1$ branch points and ramification type $(3, \ldots, 3)$.

(b) Suppose that one of the following holds:

(i) $p \geq 7$ and $n \geq \max (6+g, 2 g+1)$,

(ii) $p=3$ and $n \geq \max (7,6+g, 2 g+1)$, or

(iii) $p=5, n \geq \max (7+g, 2 g+1)$ and $n+g$ is odd.

Then we may choose $f$ with $r=g+n-1$ branch points and ramification type $(2-2, \ldots, 2-2)$.

(c) If $p=2, n \geq \max (g+3,2 g+1)$ and $n+g$ is odd, then we may choose $f$ with $r=g+n-1$ branch points and ramification type $(3, \ldots, 3)$.

(d) If $p=2, n \geq \max (g+6,2 g+3)$ and $n+g$ is even, we may choose $f$ with $r=g+n-3$ branch points, and ramification type $(5,3-3,3, \ldots, 3)$.

Proof. We start with two observations. First, to prove the theorem we may extend the base field $k$ by an arbitrary algebraically closed field extension $k^{\prime} / k$. Indeed, if we can show that the curve $X \otimes k^{\prime}$ admits a rational function with certain properties, then a standard specialization argument yields the existence of a rational function on $X$ with the same properties. Second, by the irreducibility of $\mathcal{M}_{g}$, a generic curve of a given genus is unique, up to isomorphism and extension of the base field. We may hence speak about the generic curve of genus $g$. These two observations will allow us to prove the theorem by induction on the pair $(g, n)$, using Proposition 5 .

We will discuss the induction procedure in detail for part (a). For parts (b)-(d), we will only give the necessary modifications.

Suppose that $p \neq 2,3$. For $g=0$, Theorem 10(a) says the same as Proposition 7(a). Fix an integer $g^{\prime} \geq 1$ and suppose that we have already proved Theorem 10(a) for all pairs $(g, n)$ with $g<g^{\prime}$. We have to show that Theorem 10(a) holds for all pairs $\left(g^{\prime}, n^{\prime}\right)$ with $n^{\prime} \geq \max \left(g^{\prime}+3,2 g^{\prime}+1\right)$. Write $g^{\prime}=g+1$ and $n^{\prime}=n+1$. Let $X$ be the generic curve of genus $g$ over $k$. By the induction hypothesis, there exists a tame rational function $f: X \rightarrow \mathbb{P}^{1}$ of degree $n$ with alternating monodromy, $r=g+n-1$ branch points and ramification type $(3, \ldots, 3)$. We also have $n>2 g+1$ and hence $r>3 g$. Therefore, by Proposition 5, the generic curve $X^{\prime}$ of genus $g^{\prime}$ admits a tame rational function $f^{\prime}: X^{\prime} \rightarrow \mathbb{P}^{1}$ of degree $n^{\prime}$, with monodromy group $A_{n^{\prime}}$ and ramification type $(3, \ldots, 3)$. In other words, Theorem 10 (a) holds for the pair $\left(g^{\prime}, n^{\prime}\right)$. This completes the proof of (a). 
The proof of (b) is almost the same. Note that for $g=0$ the statement of Theorem 10(b) reduces again to the statement of Proposition 7(b). The only problem occurs for $p=3$ and the pair $\left(g^{\prime}, n^{\prime}\right)=(1,7)$, because Proposition 7(b) says nothing about the case $(g, n)=(0,6)$; see also Remark 9(ii). However, in the proof of Proposition 7(b) we did construct a tame rational function $f: \mathbb{P}^{1} \rightarrow \mathbb{P}^{1}$ of degree 6 , with 5 branch points and ramification type $(2-2, \ldots, 2-2)$. We showed that its monodromy group is isomorphic to $\mathrm{PSL}_{2}(5) \cong A_{5}$. If we apply the construction underlying the proof of Proposition 5 to this cover, then we obtain a tame rational function $f^{\prime}: X^{\prime} \rightarrow \mathbb{P}^{1}$ on the generic curve of genus one of degree 7 , with ramification type $(2-2, \ldots, 2-2)$. Its monodromy group is a transitive subgroup of $A_{7}$ and contains a subgroup isomorphic to $A_{5}$. One checks, e.g. using GAP, that this group must be $A_{7}$. This settles the case $(p, g, n)=(3,1,7)$ and completes the proof of $(b)$.

The proof of (c) and (d) is again similar. The only difference is that we have a different bound on $n$ in (d). However, the induction procedure used in the proof of (a) and (b) goes through.

3.3. Open problems in characteristic 2 and 3 . As we mentioned in the introduction, our main result (Theorem 10) is optimal for $p \neq 2,3$ (in a certain sense). However, in characteristic 2 and 3 there remain a number of cases which we could not handle with the methods of the present paper. We leave these remaining cases as problems for the interested reader.

Problem 11. Let $X$ be the generic curve of genus 1 in characteristic 2. Show that there exists a tame rational function $f: X \rightarrow \mathbb{P}^{1}$ of degree 5 with monodromy group $A_{5}$ and ramification type $(3,3,3,3,3)$.

Let $g: E \rightarrow \mathbb{P}^{1}$ be the tame cyclic Galois cover of degree 3 with three branch points. Note that, in characteristic 2, the curve $E$ is the unique supersingular elliptic curve. (In characteristic 0, $E$ is an elliptic curve with complex multiplication by $\mathbb{Z}\left[\zeta_{3}\right]$.) Applying the construction underlying the proof of Proposition 4, we obtain a tame cover $f: X \rightarrow \mathbb{P}^{1}$ of degree 5 with monodromy group $A_{5}$, ramification type $(3,3,3,3,3)$ and with generic branch points. By the Riemann-Hurwitz theorem, $X$ has genus 1 . We believe but have not been able to show that $X$ is generic. The problem is the following. By construction, the cover $f: X \rightarrow \mathbb{P}^{1}$ is the generic fibre of a family of covers over a two-dimensional base. On a sublocus of codimension one of the base, this family degenerates to the cover $g: E \rightarrow \mathbb{P}^{1}$. In particular, over this sublocus the top curve of the cover is isotrivial. But it is not clear whether $X$ is isotrivial or generic.

Note that the characteristic 0 version of Problem 11 is solved in the paper [Fried et al. 2001], using the same construction as above. The proof uses the braid action to show the $X$ is not isotrivial. In fact, the case $(g, n)=(1,5)$ is the only case 
where the braid action enters into the proof of the main result of [Magaard and Völklein 2004] in an essential way.

Remark 12. At the moment, we do not know whether the generic curve of genus $g$ in characteristic 2 admits a tame rational function of degree $n$ with alternating monodromy, in each of the following cases:

(i) $(g, n)=(1,5)$,

(ii) $g \geq 3$ is odd and $n=2 g+1$,

(iii) $g \geq 2$ is even and $n=2 g+2$.

If Problem 11 (which corresponds to case (i)) had a positive solution, then also cases (ii) and (iii) would be solved, as one can see from the induction procedure in the proof of Theorem 10. If this were the case, we could omit the condition that $n+g$ is odd in the statement of Theorem 10(c) (but we would have to add the condition that $(g, n) \neq(0,4))$. This would make Theorem 10(d) superfluous, and would give an "optimal" result even in characteristic 2 .

In characteristic 3 , the existence of tame rational functions with alternating monodromy is open in the following (finite) list of cases:

$$
(g, n)=(1,5),(1,6),(2,5),(2,6),(2,7),(3,7),(3,8),(4,9) .
$$

\section{Acknowledgements}

The authors thank Gerhard Frey for stimulating this work and Helmut Völklein and the referee for useful comments on an earlier version of this manuscript.

\section{References}

[Artebani and Pirola 2003] M. Artebani and P. Pirola, "Algebraic functions with even monodromy", preprint, 2003. math.AG/0312025

[Bouw and Pries 2003] I. I. Bouw and R. J. Pries, "Rigidity, reduction, and ramification", Math. Ann. 326:4 (2003), 803-824. MR 2005b:14058 Zbl 1029.14010

[Deligne and Mumford 1969] P. Deligne and D. Mumford, "The irreducibility of the space of curves of given genus”, Inst. Hautes Études Sci. Publ. Math. 36 (1969), 75-109. MR 41 \#6850 Zbl 0181.48803

[Fried and Völklein 1991] M. D. Fried and H. Völklein, "The inverse Galois problem and rational points on moduli spaces”, Math. Ann. 290:4 (1991), 771-800. MR 93a:12004 Zbl 0763.12004

[Fried et al. 2001] M. Fried, E. Klassen, and Y. Kopeliovich, "Realizing alternating groups as monodromy groups of genus one covers", Proc. Amer. Math. Soc. 129:1 (2001), 111-119. MR 2002a: $30068 \mathrm{Zbl} 0978.30026$

[Fulton 1969] W. Fulton, "Hurwitz schemes and irreducibility of moduli of algebraic curves", Ann. of Math. (2) 90 (1969), 542-575. MR 41 \#5375 Zbl 0194.21901

[Grothendieck 1971] A. Grothendieck (editor), Revêtements étales et groupe fondamental (Séminaire de Géométrie Algébrique du Bois Marie 1960-1961 = SGA 1), edited by A. Grothendieck, Lecture Notes in Mathematics 224, Springer, Berlin, 1971. MR 50 \#7129 Zbl 0572.55016 
[Harbater and Stevenson 1999] D. Harbater and K. F. Stevenson, "Patching and thickening problems", J. Algebra 212:1 (1999), 272-304. MR 2000b:14004 Zbl 0944.14013

[Hartshorne 1977] R. Hartshorne, Algebraic geometry, Graduate Texts in Math. 52, Springer, New York, 1977. MR 57 \#3116 Zbl 0367.14001

[Huppert 1967] B. Huppert, Endliche Gruppen, I, Grundlehren der Mathematischen Wissenschaften 134, Springer, Berlin, 1967. MR 37 \#302 Zbl 0217.07201

[Knudsen 1983] F. F. Knudsen, "The projectivity of the moduli space of stable curves, II: The stacks $M_{g, n}$, Math. Scand. 52:2 (1983), 161-199. MR 85d:14038a Zbl 0544.14020

[Magaard and Völklein 2004] K. Magaard and H. Völklein, "The monodromy group of a function on a general curve”, Israel J. Math. 141 (2004), 355-368. MR 2005e:14047 Zbl 02114658

[Saïdi 1997] M. Saïdi, "Revêtements modérés et groupe fondamental de graphes de groupes", Compositio Math. 107:3 (1997), 319-338. MR 99f:14025 Zbl 0929.14016

[Schröer 2003] S. Schröer, "Curves with only triple ramification", Ann. Inst. Fourier (Grenoble) 53:7 (2003), 2225-2241. MR 2005d:14043 Zbl 1057.14035

[Serre 1992] J.-P. Serre, Topics in Galois theory, Research Notes in Mathematics 1, Jones and Bartlett, Boston, 1992. MR 94d:12006 Zbl 0746.12001

[Wewers 1998] S. Wewers, Construction of Hurwitz spaces, Ph.D. thesis, Univ. Essen, 1998, Available at http://www.math.uni-bonn.de/people/wewers.

[Wewers 1999] S. Wewers, "Deformation of tame admissible covers of curves", pp. 239-282 in Aspects of Galois theory (Gainesville, FL, 1996), edited by H. Völklein et al., London Math. Soc. Lecture Note Ser. 256, Cambridge Univ. Press, Cambridge, 1999. MR 2001b:14048 Zbl 0995.14008

Received March 1, 2004. Revised September 5, 2004.

\title{
STEFAN WEWERS \\ MATHEMATISCHES INSTITUT \\ UNIVERSITÄT BONN \\ BERINGSTRASSE 1 \\ 53115 BONN \\ GERMANY
}

wewers@math.uni-bonn.de

\author{
IRENE I. BOUW \\ MATHEMATISCHES INSTITUT \\ HEINRICH-HEINE-UNIVERSITÄT \\ UNIVERSITÄTSSTRASSE 1 \\ 40225 DÜSSELDORF \\ GERMANY \\ bouw@math.uni-duesseldorf.de
}

